\title{
On a numerical flux for the pedestrian flow equations*
}

\author{
P. KUBERA AND J. FELCMAN
}

\begin{abstract}
:
The pedestrian flow equations are formulated as the hyperbolic problem with a source term, completed by the eikonal equation yielding the desired direction of the pedestrian velocity. The operator splitting consisting of successive discretization of the eikonal equation, ordinary differential equation with the right hand side being the source term and the homogeneous hyperbolic system is proposed. The numerical flux of the Vijayasundaram type is proposed for the finite volume solution of the hyperbolic problem. The Vijayasundaram numerical flux, originally proposed for the hyperbolic problems possessing the homogeneity property is extended for pedestrian flow, where the homogeneity property is lost. The application of the proposed numerical flux is demonstrated on the physically relevant problem.
\end{abstract}

Mathematics Subject Classification 2000: 65M12, 65M50, 35k60, 76M10, 76M25

General Terms: Pedestrian flow modelling

Additional Key Words and Phrases: pedestrian flow, hyperbolic problems, finite volume method, numerical flux of Vijayasundaram type

\section{INTRODUCTION}

We deal with the computer simulation of the movement of pedestrians crowds. A macroscopic model is taken into account. It describes the pedestrian flow consisting of the continuity equation and momentum equation of fluid dynamics. Specifying the social and pressure forces in the momentum equation, we get the first order hyperbolic system of partial differential equations with a source term. Another model, namely a microscopic one, is widely used. We refer to [Bellomo and Dogbé 2008] for scaling and related representation problems. The reader finds the explanation of the macroscopic approach (the state is described by locally averaged quantities) and microscopic (the models are generally stated as a system of $N$ ordinary differential equations, where $N$ is the number of individuals) here. The microscopic model is deeply studied in [Dridi 2015], yielding a comprehensive approach to model a huge number of pedestrians and to simulate high density crowd behaviour in overcrowding places, e.g. sport, concert and pilgrimage places.A useful

${ }^{*}$ The research of the first author was supported by the project Grant Severočeských dolů Chomutov, the research of the second author was supported by the project 13-00522S of the Czech Science Foundation. The authors acknowledge this support. 
insight into macroscopic and microscopic models can be found in [Marno 2002]

The splitting technique is applied which leads to a combination of the finite volume method for the hyperbolic problem with the numerical solution of the system of ordinary differential equations. Additionally, the solution of the so-called eikonal equation plays an important role here. Such a solution determines the density dependent direction of pedestrian motion.

We use the macroscopic model of Payne-Whitham type, see e.g. [Twarogowska et al. 2013]. In this model pedestrians are considered as an intelligent fluid which movement is influenced by the so-called social forces. The social forces model assumes that each pedestrian is trying to move in a desired direction (e.g. to the exit) with a desired velocity, where his velocity and the direction of movement depends on the density of pedestrians in his neighborhood.

Let us consider the flow of pedestrians in e.g. sport, concert and pilgrimage places. Pedestrians are supposed to occupy the two dimensional bounded domain $\Omega$ and we are interested with their movement in the time interval $(0, T)$ with $T>0$. We denote $\partial \Omega$ the boundary of $\Omega$. We suppose that the boundary $\partial \Omega=\bar{\Gamma}_{w} \cup \bar{\Gamma}_{o}$, where the disjoint subsets $\Gamma_{w}, \Gamma_{o} \in \partial \Omega$ are formed by a finite number of connected sets, open with respect to the topology on $\partial \Omega$, and the one-dimensional meas $\left(\Gamma_{o}\right)>0 . \Gamma_{w}$ and $\Gamma_{o}$ represent the walls and the exit, respectively. The pedestrian flow equations (PFE) are derived as a two dimensional sub-model of the general governing equations for the dynamics of fluids. They represent an extension to a pedestrian motion of the Payne-Whitham vehicular traffic model, further developed by Jiang to describe a crowd dynamics.

Denoting by $\rho$ the density of pedestrians in $\left[\mathrm{ped} / \mathrm{m}^{2}\right]$, by $v=\left(v_{1}, v_{2}\right)$ their velocity in $[\mathrm{m} / \mathrm{s}]$ and by $p$ the pressure in $\left[\mathrm{N} / \mathrm{m}^{2}\right]$, the model is given by the conservation of mass (continuity equation) and by the conservation of momentum. The latter is described by the equation of motion of an inviscid fluid subject to volume force given by the quantity $\mathbf{f}$ in $\left[\mathrm{m} / \mathrm{s}^{2}\right]$. Then the system of PFE reads

$$
\begin{aligned}
& \frac{\partial \rho}{\partial t}+\operatorname{div}(\rho v)=0, \\
& \frac{\partial \rho v}{\partial t}+\operatorname{div}(\rho v \otimes v)=\rho \mathbf{f}+\operatorname{div} \mathscr{T},
\end{aligned}
$$

where the stress tensor $\mathscr{T}$ has the form $\mathscr{T}=-p \mathbb{I}$, i.e. $\operatorname{div} \mathscr{T}=-\nabla p$. In this way we get formally the same system of conservation laws as in e.g. [Feistauer et al. 2003, page 23]. We complete system (1)-(2) by the relation for the pressure $p$ (preventing 
from overcrowding) given by the power law for isentropic gases

$$
p=p(\rho)=p_{0} \rho^{\gamma}, p_{0}>0, \gamma>1 \text {. }
$$

Further we have to specify the quantity $\mathbf{f}$ which describes the acceleration caused by internal driving forces and motivations of pedestrians. It represents a relaxation term towards a desired velocity. The quantity $\mathbf{f}$ is expressed in terms of the velocity $v$ and quantities $V=V(\rho)$ and $\mu=\mu(\rho)$ characterizing the magnitude of desired velocity and its direction, respectively. We use the relation $\mathbf{f}=\frac{1}{\tau} \rho(V \mu-v)$. See [Felcman and Kubera 2015] for more details. The formula for $V=V(\rho)$ is explicit, the quantity $\mu$ is obtained as the negative gradient of the scalar potential $\phi=\phi(\rho)$ stemming as the solution of the so called eikonal equation:

$$
\begin{array}{r}
|\nabla \phi|=c \text { in } \Omega, \\
\phi=0 \text { on } \Gamma_{o} \subset \partial \Omega,
\end{array}
$$

with the prescribed right hand side $c=c(\rho), c=1 / V$ taken from [Twarogowska et al. 2013]:

$$
V=V(\rho)=v_{\max } e^{-\alpha\left(\frac{\rho}{\rho_{\max }}\right)^{2}}
$$

with given constants $v_{\max }, \alpha$ and $\rho_{\max }$.

\section{OPERATOR SPLITTING}

One possible way for the discretization of pedestrian flow equations is the operator splitting. It is motivated by the properties of system (1)-(2), which can be treated as hyperbolic partial differential equations with source term. Following [Toro 1997, Chapter 15] we split the complete system into the reaction-like system and the hyperbolic system and discretize them separately. The coupling via the so called eikonal equation has to be taken into account. The constituent equations for the unknown vector valued function $w$ are (For details see [Felcman and Kubera 2015]):

$$
\begin{gathered}
|\nabla \phi|=c \text { in } \Omega, \\
\phi=0 \text { on } \Gamma_{o} \subset \partial \Omega, \\
\mu=-\frac{\nabla \phi}{|\nabla \phi|},
\end{gathered}
$$




$$
\begin{gathered}
\frac{\partial w}{\partial t}=\left(0, \frac{1}{\tau} \rho(V \mu-v)\right)^{\mathrm{T}} \\
\frac{\partial w}{\partial t}+\sum_{s=1}^{2} \frac{\partial f_{s}(w)}{\partial x_{s}}=0
\end{gathered}
$$

where $f_{s}(w)=\left(\rho v_{s}, \rho v_{1} v_{s}+\delta_{s 1} p_{0} \rho^{\gamma}, \rho v_{2} v_{s}+\delta_{s 2} p_{0} \rho^{\gamma}\right)^{\mathrm{T}}$ and $\rho$ is the first component of vector valued function $w$ and density functions $V$ and $c$ are given by explicit relations. The quantity $w=\left(w_{1}, w_{2}, w_{3}\right)^{\mathrm{T}} \equiv(\rho, q)^{\mathrm{T}} \equiv(\rho, \rho v)^{\mathrm{T}} \equiv\left(\rho, \rho v_{1}, \rho v_{2}\right)^{\mathrm{T}}$.

Remark 2.1. If in the operator splitting (7)- (11) equation (10) is neglected and equation (11) is replaced by

$$
\frac{\partial w}{\partial t}+\sum_{s=1}^{2} \frac{\partial f_{s}(w)}{\partial x_{s}}=s(w)
$$

where

$$
s(w)=\left(0, \frac{1}{\tau} \rho(V \mu-v)\right)^{\mathrm{T}},
$$

then system (1)-(5) is equivalent to equations (7)-(9), (12).

Equation (7) is discretized by the finite element method (FEM), whereas system (11) is discretized by the finite volume method (FVM). System (10) is basically a system of ordinary differential equations and it can be solved e.g. by a Runge-Kutta method. Here we only concentrate on the discretization of system (11) by the finite volume method introducing the new numerical flux of Vijayasundaram type, which is the novelty of the paper. The discretization of other parts of operator splitting is described in [Felcman and Kubera 2015].

\subsection{The properties of the pedestrian flow equations}

It is known from the theory of hyperbolic systems of type (11) that the homogeneity, the hyperbolicity and the rotational invariance properties allow us to construct effective numerical fluxes for the finite volume solution of the related problem. The purpose of this paper is to show, that despite that the pedestrian flow equations formulated as the hyperbolic system do not possess the homogeneity property, we can design the numerical flux of Vijayasundaram type, originally constructed for the Euler equations of motion and based on the homogeneity property of the Euler equations. Its competitiveness to other numerical fluxes will be demonstrated on the numerical examples. 
2.1.1 Homogeneity. The homogeneity property known to be formulated as

$$
f_{s}(w)=\mathbb{A}_{s}(w) w
$$

where $\mathbb{A}_{s}(w)$ is the Jacobi matrix of the flux $f_{s}, s=1,2$, is for the pedestrian flow equations replaced by the relations

$$
f_{s}(w)=\mathbb{A}_{s}(w) w-\left(\begin{array}{c}
0 \\
p_{0} \rho^{\gamma} \delta_{s 1} \\
p_{0} \rho^{\gamma} \delta_{s 2}
\end{array}\right), \quad s=1,2 .
$$

2.1.2 Rotational invariance of the pedestrian flow equations. Let us consider an orthogonal coordinate transformation

$$
\tilde{x}=\mathbb{Q}_{0} x+\tilde{\sigma}
$$

where $\mathbb{Q}_{0}^{T} \mathbb{Q}_{0}=\mathbb{Q}_{0} \mathbb{Q}_{0}^{T}=I$ and $\tilde{\sigma} \in \mathbb{R}^{2}$. System (11) is rotationally invariant, i.e. a function $w=w(x, t)$ solves (in classical sense, for simplicity) (11) if and only if the function $\tilde{w}=\tilde{w}(\tilde{x}, t)=\mathbb{Q} w\left(\mathbb{Q}_{0}^{-1}(\tilde{x}-\tilde{\sigma}), t\right)$ solves the transformed system

$$
\frac{\partial \tilde{w}}{\partial t}+\sum_{s=1}^{2} \frac{\partial}{\partial \tilde{x}_{s}} f_{s}(\tilde{w})=0
$$

as well. The matrix $\mathbb{Q}$ is defined in (18). The rotational invariance can be also stated as a property of the fluxes $f_{s}$.

Definition 2.2. Let us denote

$$
\mathscr{P}(w, n)=\sum_{s=1}^{2} n_{s} f_{s}(w)
$$

the flux (of the quantity $w$ ) in the direction $n=\left(n_{1}, n_{2}\right)^{T}$.

The flux is rotationally invariant, if

$$
\mathscr{P}\left(\mathbb{Q} w, \mathbb{Q}_{0} n\right)=\mathbb{Q} \mathscr{P}(w, n), \quad \mathbb{Q}=\left(\begin{array}{cc}
1 & 0 \\
0 & \mathbb{Q}_{0}
\end{array}\right) \in \mathbb{R}^{3 \times 3} .
$$

LEMMA 2.3. The flux for the pedestrian flow system (11) is rotationally invariant.

PROOF. Let us prove (18) directly. Recall that $w=(\rho, \rho v)^{T}=\left(\rho, \rho v_{1}, \rho v_{2}\right)^{T}$ and 
$q=\rho v$.

$$
\begin{aligned}
\mathscr{P}(w, n) & =n_{1}\left(\begin{array}{c}
q_{1} \\
\rho^{-1} q_{1}^{2}+p_{0} \rho^{\gamma} \\
\rho^{-1} q_{1} q_{2}
\end{array}\right)+n_{2}\left(\begin{array}{c}
q_{2} \\
\rho^{-1} q_{1} q_{2} \\
\rho^{-1} q_{2}^{2}+p_{0} \rho^{\gamma}
\end{array}\right) \\
& =\frac{n^{T} q}{\rho}\left(\begin{array}{l}
\rho \\
q
\end{array}\right)+p_{0} \rho^{\gamma}\left(\begin{array}{l}
0 \\
n
\end{array}\right), \\
\mathscr{P}\left(\mathbb{Q} w, \mathbb{Q}_{0} n\right) & =\frac{\left(\mathbb{Q}_{0} n\right)^{T} \mathbb{Q}_{0} q}{\rho}\left(\begin{array}{c}
\rho \\
\mathbb{Q}_{0} q
\end{array}\right)+\frac{1}{2} g \rho^{2}\left(\begin{array}{c}
0 \\
\mathbb{Q}_{0} n
\end{array}\right) \\
& =\frac{n^{T} q}{\rho} \mathbb{Q}\left(\begin{array}{l}
\rho \\
q
\end{array}\right)+p_{0} \rho^{\gamma} \mathbb{Q}\left(\begin{array}{l}
0 \\
n
\end{array}\right)=\mathbb{Q} \mathscr{P}(w, n) .
\end{aligned}
$$

This proves (18).

The consequence of relation (18) is the following

LEMmA 2.4. Let $w \in D$ and $n \in \mathbb{R}^{2},|n|=1$. Then

$$
\mathscr{P}(w, n)=\mathbb{Q}^{-1} f_{1}(\mathbb{Q} w), \quad \mathbb{Q}_{0}=\mathbb{Q}_{0}(n)=\left(\begin{array}{cc}
n_{1} & n_{2} \\
-n_{2} & n_{1}
\end{array}\right) .
$$

Proof. The matrix $\mathbb{Q}_{0}$ is orthogonal and it holds $\mathbb{Q}_{0} n=(1,0)^{T}$ (matrix-vector product). It follows from (18)

$$
\begin{aligned}
\mathscr{P}(w, n) & =\mathbb{Q}^{-1} \mathscr{P}\left(\mathbb{Q} w, \mathbb{Q}_{0} n\right)=\mathbb{Q}^{-1}[\underbrace{\left(\mathbb{Q}_{0} n\right)_{1}}_{=1} f_{1}(\mathbb{Q} w)+\underbrace{\left(\mathbb{Q}_{0} n\right)_{2}}_{=0} f_{2}(\mathbb{Q} w)] \\
& =\mathbb{Q}^{-1} f_{1}(\mathbb{Q} w) .
\end{aligned}
$$

2.1.3 Hyperbolicity of the pedestrian flow equations. Let us set $a=\sqrt{\gamma \frac{p}{\rho}}$.

LEMMA 2.5. The matrix

$$
\mathbb{A}_{1}(w)=\left(\begin{array}{ccc}
0 & 1 & 0 \\
-h^{-2} q_{1}^{2}+a^{2} & 2 h^{-1} q_{1} & 0 \\
-h^{-2} q_{1} q_{2} & h^{-1} q_{2} & h^{-1} q_{1}
\end{array}\right)=\left(\begin{array}{ccc}
0 & 1 & 0 \\
-v_{1}^{2}+a^{2} & 2 v_{1} & 0 \\
-v_{1} v_{2} & v_{2} & v_{1}
\end{array}\right)
$$

is the Jacobi matrix of the function $f_{1}$.

LEMMA 2.6. The matrix $\mathbb{A}_{1}(w)$ has the eigenvalues

$$
\tilde{\lambda}_{1}(w)=v_{1}-a, \quad \tilde{\lambda}_{2}(w)=v_{1}, \quad \tilde{\lambda}_{3}(w)=v_{1}+a .
$$


The corresponding eigenvectors have the form

$$
r_{1}(w)=\left(\begin{array}{c}
1 \\
v_{1}-a \\
v_{2}
\end{array}\right), \quad r_{2}(w)=\left(\begin{array}{l}
0 \\
0 \\
1
\end{array}\right), \quad r_{3}(w)=\left(\begin{array}{c}
1 \\
v_{1}+a \\
v_{2}
\end{array}\right) .
$$

The eigenvectors $r_{1}(w), \ldots, r_{3}(w)$ of the matrix $\mathbb{A}_{1}(w)$ are linearly independent for each $w \in D$ and, hence, they form a basis in the space $\mathbb{R}^{3}$. Denoting by $\tilde{\mathbb{T}}(w)$ the matrix having the vectors $r_{1}(w), \ldots, r_{3}(w)$ as its columns, it is clear that $\tilde{\mathbb{T}}(w)$ is nonsingular and

$$
\mathbb{A}_{1}(w) \tilde{\mathbb{T}}(w)=\tilde{\mathbb{T}}(w) \tilde{\Lambda} \backslash(w),
$$

where

$$
\tilde{\Lambda} \backslash(w)=\operatorname{diag}\left(\tilde{\lambda}_{1}(w), \ldots, \tilde{\lambda}_{3}(w)\right)
$$

which is the diagonal matrix with diagonal entries $\tilde{\lambda}_{1}(w), \ldots, \tilde{\lambda}_{3}(w)$. Hence,

$$
\tilde{\mathbb{T}}^{-1}(w) \mathbb{A}_{1}(w) \tilde{\mathbb{T}}(w)=\tilde{\Lambda}(w) .
$$

This means that the matrix $\mathbb{A}_{1}$ is diagonalizable.

THEOREM 2.7. The pedestrian flow equations (12) with zero right hand side form a diagonally hyperbolic system in the sense of Definition 2.3 from [Feistauer et al. 2003, page 59]

PROOF. It can be carry out analogously as for the Euler equations, see [Feistauer et al. 2003, Theorem 3.6, page 108]

\section{FINITE VOLUME METHOD}

A standard finite volume method for system (11) [Feistauer et al. 2003], [Eymard et al. 2000] can be applied for finding the value of the approximate solution $w_{i}^{k}$ on the finite volume $D_{i}$ at time $t_{k},\left.w\left(\cdot, t_{k}\right)\right|_{D_{i}} \approx w_{i}^{k}=$ const.:

$$
w_{i}^{k+1}=w_{i}^{k}-\frac{\tau^{k}}{\left|D_{i}\right|} \sum_{j \in S(i)}\left|\Gamma_{i j}\right| H\left(w_{i}^{k}, w_{j}^{k}, n_{i j}\right) .
$$

Such a scheme is based on the construction of the finite volume mesh $\mathscr{D}_{h}=\left\{D_{i}\right\}_{i \in J}$, where $J \subset Z^{+}=\{0,1, \ldots\}$ is an index set and $h>0$. Here $D_{i}, i \in J$, are closed polygons with mutually disjoint interiors. For two neighbours $D_{i}, D_{j} \in \mathscr{D}_{h}$ we set $\Gamma_{i j}=\partial D_{i} \cap \partial D_{j}=\Gamma_{j i}$. We denote by $\left|D_{i}\right|$ or $\left|\Gamma_{i j}\right|$ the 2-dimensional measure of $D_{i}$ or the (1)-dimensional measure of $\Gamma_{i j}$, respectively. 
We construct a partition $0=t_{0}<t_{1}<\ldots$ of the time interval $[0, T]$ and denote by $\tau_{k}=t_{k+1}-t_{k}$ the time step between $t_{k}$ and $t_{k+1}$. Scheme (23) represents an algorithm for finding the approximations of the integral averages $\int_{D_{i}} w\left(x, t_{k}\right) d x /\left|D_{i}\right|$ of the quantity $w$ over the finite volume $D_{i}$ at time instant $t_{k}$. Further, we approximate the flux $\sum_{s=1}^{N} f_{s}(w)\left(n_{i j}\right)_{s}$ of the quantity $w$ through the face $\Gamma_{i j}$ in the direction $n_{i j}$ with the aid of a numerical flux $H\left(w_{i}^{k}, w_{j}^{k}, n_{i j}\right)$. The novelty of this paper is the derivation of the original numerical flux $H$ of Vijayasundaram type for the pedestrian flow equations. Method (23) is equipped with initial conditions $w_{i}^{0}, i \in J$, defined as integral averages of $w^{0}$ under the assumption that the function $w^{0}$ is locally integrable: $w^{0} \in L_{\mathrm{loc}}^{1}(\Omega)^{3}$.

We consider the finite volume solution as piecewise constant vector-valued functions $\mathbf{w}_{\mathscr{D} k}^{k}, k=0,1, \ldots$, defined almost everywhere in the computational domain $\Omega$ so that $\left.\mathbf{w}_{\mathscr{D} k}^{k}\right|_{D_{i}} ^{\circ}=w_{i}^{k}$ for all $i \in J$, where $\stackrel{\circ}{D_{i}}$ is the interior of $D_{i}$, i.e. $\stackrel{\circ}{D_{i}}=D_{i} \backslash \partial D_{i}$, and $w_{i}^{k}$ are obtained from the finite volume formula (23). The index $k$ in $\mathscr{D}^{k}$ indicates that the used mesh could be time dependent. The function $\mathbf{w}_{\mathscr{D} k}^{k}$ is the approximate solution at time $t=t_{k}$ on the finite volume mesh $\mathscr{D}^{k}$. The vector $w_{i}^{k}$ is the value of the approximate solution on the finite volume $D_{i} \in \mathscr{D}^{k}$ at time $t_{k}$.

We are interested in Godunov type methods. Thanks to the rotational invariance of system under consideration (see relation (19)), the numerical flux $H(\cdot, \cdot, \cdot)$ approximating the flux $\left.\sum_{s=1}^{2} n_{s} f_{s}(w)\right|_{\Gamma_{i j}}$ reduces to a Godunov numerical flux $g(\cdot, \cdot)$ for a system with one space dimension via relation

$$
H\left(w_{L}, w_{R}, n\right)=\mathbb{Q}^{-1} g\left(\mathbb{Q} w_{L}, \mathbb{Q} w_{R}\right),
$$

where $w_{L}:=w_{i}^{k}, w_{R}:=w_{j}^{k}, n:=n_{i j}$ and the matrix $\mathbb{Q}=\mathbb{Q}(n)$ is defined in (18), (19). This relation follows from the approximation of $\tilde{w}=\mathbb{Q} w$ on $\Gamma_{i j}$ by the similarity solution $\tilde{w}_{R S}\left(0, \tilde{w}_{L}, \tilde{w}_{R}\right)$ (RS stands for Riemann solution) of the Riemann problem

$$
\frac{\partial \tilde{w}}{\partial t}+\frac{\partial f_{1}(\tilde{w})}{\partial \tilde{x}_{1}}=0, \quad \tilde{w}\left(\tilde{x}_{1}, 0\right)= \begin{cases}\tilde{w}_{L}=\mathbb{Q} w_{L}, & \tilde{x}_{1}<0 \\ \tilde{w}_{R}=\mathbb{Q} w_{R}, & \tilde{x}_{1}>0 .\end{cases}
$$

Using (19) we proceed in the following way

$$
\begin{array}{r}
\left.\sum_{s=1}^{N} n_{s} f_{s}\left(w\left(\cdot, t_{k}\right)\right)\right|_{\Gamma_{i j}} \approx \mathbb{Q}^{-1} f_{1}(\overbrace{\tilde{w}_{R S}\left(0, \tilde{w}_{L}, \tilde{w}_{R}\right)}^{\mathbb{Q} w}) \\
\approx \mathbb{Q}^{-1} g\left(\tilde{w}_{L}, \tilde{w}_{R}\right)=: H\left(w_{i}^{k}, w_{j}^{k}, n_{i j}\right) .
\end{array}
$$

The method is fully specified by the choice of the Riemann numerical flux $g(\cdot, \cdot)$ 
(approximate Riemann solver), which is a suitable approximation of the Godunov numerical flux $f_{1}\left(\tilde{w}_{R S}(0, \cdot, \cdot)\right)$ (exact Riemann solver). The numerical fluxes can be also derived from finite difference approximations to (12). This approach leads to central schemes, e.g. to the Lax-Friedrichs flux. Godunov type methods are based on exact or approximate solvers of the Riemann problem for the one-dimensional version of (12), e.g. the VFRoe fluxes of [Gallouët et al. 2003].

Here, we present the numerical flux, based on well-known numerical fluxes context of the compressible Euler equations (see [Feistauer et al. 2003]). It exploits the integral forms of the Godunov numerical flux

$$
g\left(w_{L}, w_{R}\right) \approx f_{1}\left(w_{L}\right)+\int_{\langle\eta\rangle} \mathbb{A}_{1}^{-}(w) d w
$$

or

$$
g\left(w_{L}, w_{R}\right) \approx f_{1}\left(w_{R}\right)-\int_{\langle\eta\rangle} \mathbb{A}_{1}^{+}(w) d w
$$

and their combination

$$
g\left(w_{L}, w_{R}\right):=\frac{1}{2}\left\{f_{1}\left(w_{L}\right)+f_{1}\left(w_{R}\right)-\int_{\langle\eta\rangle}\left|\mathbb{A}_{1}(w)\right| d w\right\} .
$$

Here $\eta$ is a suitable integration path connecting states $w_{L}$ and $w_{R}$ and matrices $\mathbb{A}_{1}^{ \pm}=\tilde{\mathbb{T}} \operatorname{diag}\left(\tilde{\lambda}_{1}^{ \pm}, \tilde{\lambda}_{2}^{ \pm}, \tilde{\lambda}_{3}^{ \pm}\right) \tilde{\mathbb{T}}^{-1}, \quad \tilde{\lambda}_{i}^{ \pm}$being the positive and negative parts of eigenvalues of the Jacobi matrix $\mathbb{A}_{1}$ of the flux $f_{1}$ and $\tilde{\mathbb{T}}$ being the matrix that diagonalize $\mathbb{A}_{1},\left|\mathbb{A}_{1}(w)\right|=\mathbb{A}_{1}^{+}(w)-\mathbb{A}_{1}^{-}(w)$. The integrals above do not make sense in general. However, these formulae can be used for the definition of numerical schemes in such a way that the integrals are evaluated with the aid of some numerical quadratures or computed with the use of suitable integration path in the state space. The motivation for the above integral forms of Godunov numerical fluxes can be found in [Feistauer et al. 2003, Sec. 3.2.15-3.2.16, page 158].

\subsection{Numerical Flux of Vijayasundaram Type}

The Vijayasundaram numerical flux was originally derived for the Euler equations in [Vijayasundaram 1982]. In [Feistauer et al. 2003, page 163] the Vijayasundaram flux for the Euler equations was derived from integral form (27) using the first order numerical quadrature and the homogeneity (14) of the Euler flux $f_{1}$.

Since the pedestrian flow equations do not possess property (14), we cannot simply adopt the Vijayasundaram numerical flux for the Euler equations. One way how to overcome this obstacle is to use the fact, that for the pedestrian flow equations there is a matrix $\mathbb{B}$ such that $f_{1}(w)=\mathbb{B}(w) w$. Here we deal with another approach, which 
is the novelty of this paper. The construction of the numerical flux is still an open question, namely for the case, where the density $\rho$ vanishes. Without doubts the higher order methods are demanded (e.g. ADER technique of Toro, or the approach from [Furmanek et al. 2009]) as well.

Here we propose the new numerical flux

$$
\begin{aligned}
g_{1}\left(w_{L}, w_{R}\right) & =\mathbb{A}_{1}^{+}\left(\frac{w_{L}+w_{R}}{2}\right) w_{L}+\mathbb{A}_{1}^{-}\left(\frac{w_{L}+w_{R}}{2}\right) w_{R} \\
& -p_{0}\left(\frac{\rho_{L}+\rho_{R}}{2}\right)^{\gamma}\left(\begin{array}{l}
0 \\
1 \\
0
\end{array}\right) .
\end{aligned}
$$

It is obtained from (27) using the following approximation

$$
\begin{gathered}
\frac{1}{2}\left(f_{1}\left(w_{L}\right)+f_{1}\left(w_{R}\right)\right) \approx f_{1}\left(\frac{w_{L}+w_{R}}{2}\right)= \\
=\mathbb{A}_{1}\left(\frac{w_{L}+w_{R}}{2}\right)\left(\frac{w_{L}+w_{R}}{2}\right)-p_{0}\left(\frac{\rho_{L}+\rho_{R}}{2}\right)^{\gamma}\left(\begin{array}{l}
0 \\
1 \\
0
\end{array}\right)
\end{gathered}
$$

and the numerical quadrature

$$
\int_{w_{L}}^{w_{R}}\left|\mathbb{A}_{1}\right| d w \approx\left|\mathbb{A}_{1}\left(\frac{w_{L}+w_{R}}{2}\right)\right|\left(w_{R}-w_{L}\right) .
$$

THEOREM 3.1. The numerical flux $H$ defined in (24) with $g\left(w_{L}, w_{R}\right):=g_{1}\left(w_{L}, w_{R}\right)$ from $(28)$ is consistent, i.e. $H(w, w, n)=\sum_{s=1}^{2} n_{s} f_{s}(w)$.

PROOF. It holds

$$
\begin{aligned}
\mathbb{Q}^{-1} g_{1}(w, w) & =\mathbb{Q}^{-1}\left[\mathbb{A}_{1}^{+}(\mathbb{Q} w) \mathbb{Q} w+\mathbb{A}_{1}^{-}(\mathbb{Q} w) \mathbb{Q} w-p_{0} \rho^{\gamma}\left(\begin{array}{l}
0 \\
1 \\
0
\end{array}\right)\right] \\
& =\mathbb{Q}^{-1}\left[\mathbb{A}_{1}(\mathbb{Q} w) \mathbb{Q} w-p_{0} \rho^{\gamma}\left(\begin{array}{l}
0 \\
1 \\
0
\end{array}\right)\right] \\
& =\mathbb{Q}^{-1} f_{1}(\mathbb{Q} w)=\sum_{s=1}^{2} n_{s} f_{s}(w) .
\end{aligned}
$$


To prove the conservation of the numerical flux $H$ we first introduce the following

LEMMA 3.2. Let $\mathbb{Z}$ be the diagonal matrix, $\mathbb{Z}=\operatorname{diag}\{1,-1,-1\}$. Then it holds

$$
\begin{aligned}
& \mathbb{A}_{1}^{+}(\mathbb{Z} w)=-\mathbb{Z} \mathbb{A}_{1}^{-}(w) \mathbb{Z} \\
& \mathbb{A}_{1}^{-}(\mathbb{Z} w)=-\mathbb{Z} \mathbb{A}_{1}^{+}(w) \mathbb{Z} .
\end{aligned}
$$

Proof. Let $\tilde{\mathbb{T}}$ be the matrix, which diagonalize $\mathbb{A}_{1}$. First we transform the matrix $\mathbb{A}_{1}(\mathbb{Z} w)$ to the diagonal form. For $n=(-1,0)^{\mathrm{T}}$ we get for $\mathbb{Q}$ in $(24) \mathbb{Q}=\mathbb{Q}^{-1}=\mathbb{Z}$ and the rotational invariance property gives

$$
\sum_{s=1}^{2} n_{s} f_{s}(w)=-f_{1}(w)=\mathbb{Q}^{-1} f_{1}(\mathbb{Q} w)=\mathbb{Z} f_{1}(\mathbb{Z} w) .
$$

The derivation of $-f_{1}(w)$ with respect to $w$ yields

$$
-\mathbb{A}_{1}(w)=\mathbb{Z} \mathbb{A}_{1}(\mathbb{Z} w) \mathbb{Z}
$$

Expressing $\mathbb{A}_{1}(\mathbb{Z} w)$ from the previous equality and diagonalizing $-\mathbb{A}_{1}(w)$ we get

$$
\mathbb{A}_{1}(\mathbb{Z} w)=\mathbb{Z} \tilde{\mathbb{T}}(w)[-\Lambda \backslash(w)][\mathbb{Z} \tilde{\mathbb{T}}(w)]^{-1} .
$$

It holds for all $x \in \mathbb{R}$

$$
(-x)^{+}=-x^{-}, \quad(-x)^{-}=-x^{+}
$$

and this property holds for diagonal matrices as well. So

$$
\begin{aligned}
\mathbb{A}_{1}^{+}(\mathbb{Z} w) & \left.=\mathbb{Z} \tilde{\mathbb{T}}(w)[-\Lambda \backslash(w)]^{+}[\mathbb{Z} \tilde{\mathbb{T}}(w)]^{-1}=-\mathbb{Z} \tilde{\mathbb{T}}(w)\right) \Lambda \backslash(w)^{-}[\mathbb{Z} \tilde{\mathbb{T}}(w)]^{-1} \\
& =-\mathbb{Z} \mathbb{A}_{1}^{-}(w) \mathbb{Z} \\
\mathbb{A}_{1}^{-}(\mathbb{Z} w) & =\mathbb{Z} \tilde{\mathbb{T}}(w)[-\Lambda \backslash(w)]^{-}[\mathbb{Z} \tilde{\mathbb{T}}(w)]^{-1}=-\mathbb{Z} \tilde{\mathbb{T}}(w) \Lambda \backslash(w)^{+}[\mathbb{Z} \tilde{\mathbb{T}}(w)]^{-1} \\
& =-\mathbb{Z} \mathbb{A}_{1}^{+}(w) \mathbb{Z} .
\end{aligned}
$$

THEOREM 3.3. The numerical flux $H$ defined in (24) with $g\left(w_{L}, w_{R}\right) \quad:=g_{1}\left(w_{L}, w_{R}\right) \quad$ from (28) is conservative, i.e. $H\left(w_{L}, w_{R}, n\right)=-H\left(w_{R}, w_{L},-n\right)$.

PROOF. Let us write $H\left(w_{L}, w_{R}, n\right)$ in the form

$$
H\left(w_{L}, w_{R}, n\right)=H_{1}\left(w_{L}, w_{R}, n\right)-p_{0} H_{2}\left(w_{L}, w_{R}, n\right),
$$


where

$$
\begin{aligned}
& H_{1}\left(w_{L}, w_{R}, n\right)=\mathbb{Q}^{-1}\left[\mathbb{A}_{1}^{+}\left(\mathbb{Q} \frac{w_{L}+w_{R}}{2}\right) \mathbb{Q} w_{L}+\mathbb{A}_{1}^{-}\left(\mathbb{Q} \frac{w_{L}+w_{R}}{2}\right) \mathbb{Q} w_{R}\right], \\
& H_{2}\left(w_{L}, w_{R}, n\right)=-\left(\frac{\rho_{L}+\rho_{R}}{2}\right)^{\gamma} \mathbb{Q}^{-1}\left(\begin{array}{l}
0 \\
1 \\
0
\end{array}\right)=\left(\frac{\rho_{L}+\rho_{R}}{2}\right)^{\gamma}\left(\begin{array}{l}
0 \\
n_{1} \\
n_{2}
\end{array}\right) .
\end{aligned}
$$

It is sufficient to prove the conservation property separately for $H_{1}$ and $H_{2}$.

For $H_{1}$ we express $\mathbb{Q}=\mathbb{Q}(n)$ defined in (24) with the aid of $\mathbb{Q}(-n)$ and $\mathbb{Z}$ defined in Lemma 3.2 as

$$
\mathbb{Q}(n)=\mathbb{Z} \mathbb{Q}(-n), \quad \mathbb{Q}(n)^{-1}=\mathbb{Q}(-n)^{-1} \mathbb{Z},
$$

and we take use of Lemma 3.2.

$$
\begin{aligned}
& H_{1}\left(w_{L}, w_{R}, n\right) \\
& =\mathbb{Q}(-n)^{-1} \mathbb{Z}\left[\mathbb{A}_{1}^{+}\left(\mathbb{Z} \mathbb{Q}(-n) \frac{w_{L}+w_{R}}{2}\right) \mathbb{Z} \mathbb{Q}(-n) w_{L}\right. \\
& \left.+\mathbb{A}_{1}^{-}\left(\mathbb{Z} \mathbb{Q}(-n) \frac{w_{L}+w_{R}}{2}\right) \mathbb{Z} \mathbb{Q}(-n) w_{R}\right] \\
& =-\mathbb{Q}(-n)^{-1}\left[\mathbb{A}_{1}^{-}\left(\mathbb{Q}(-n) \frac{w_{L}+w_{R}}{2}\right) \mathbb{Q}(-n) w_{L}\right. \\
& \left.+\mathbb{A}_{1}^{+}\left(\mathbb{Q}(-n) \frac{w_{L}+w_{R}}{2}\right) \mathbb{Q}(-n) w_{R}\right] \\
& =-H_{1}\left(w_{R}, w_{L},-n\right)
\end{aligned}
$$

The conservation property of $H_{2}$ follows immediately from

$$
\begin{aligned}
H_{2}\left(w_{L}, w_{R}, n\right) & =\left(\frac{\rho_{L}+\rho_{R}}{2}\right)^{\gamma}\left(\begin{array}{c}
0 \\
n_{1} \\
n_{2}
\end{array}\right) \\
& =-\left(\frac{\rho_{R}+\rho_{L}}{2}\right)^{\gamma}\left(\begin{array}{c}
0 \\
-n_{1} \\
-n_{2}
\end{array}\right) \\
& =-H_{2}\left(w_{R}, w_{L},-n\right) .
\end{aligned}
$$




\section{NUMERICAL SOLUTION}

System (1)-(2) was solved numerically via operator splitting consisting of successive discretization of equations (7)-(11) in an iterative manner. Based on the mathematical properties of system (11), the finite volume approach was used. It was combined with a finite element framework for the solution of the eikonal equation (7). The aim is to find the value of the approximate solution $w_{i}^{k}$ on the finite volume $D_{i}$ at time $t_{k}$, $\left.w\left(\cdot, t_{k}\right)\right|_{D_{i}} \approx w_{i}^{k}=$ const.

\subsection{Operator splitting algorithm}

We discretize each equation of (7)-(11) separately.

(0) Let us consider the set $Z_{h}=\left\{\varphi_{h} \in L^{2}(\Omega) ;\left.\varphi_{h}\right|_{D}=\text { const } \forall D \in \mathscr{D}_{h}\right\}^{3}$. We start from the approximation $\mathbf{w}^{0} \in Z_{h}$ and we consider a second order Runge-Kutta method for the solution of an initial value problem for ordinary differential equations and the finite volume method for the solution of hyperbolic problems of type (11). The CFL condition for the time step $\tau_{k}$ in the finite volume method plays an important role for setting of the time step in the Runge-Kutta method. Let us assume that we have already obtained the approximation $\mathbf{w}^{k} \in Z_{h}$ at time level $t_{k}$. The transition to the next time level is carried out in four steps.

(1) The eikonal equation (7) solution: For the solution of the eikonal equation (7) we implemented the Bornemann and Rash algorithm [Bornemann and Rasch 2006]. The potential $\phi$ is approximated by the piecewise linear function $\phi_{h} \in X_{h}=\left\{\phi_{h} \in C(\bar{\Omega}) ; \phi_{h} \mid D_{i}\right.$ are piecewise linear on the triangulation $\left.\mathscr{D}_{h}\right\}$. The key point of this algorithm is the use of the Hopf-Lax formula in order to obtain the piecewise linear finite element solution of the certain local Dirichlet problem. To this end we construct the piecewise linear recovery $\rho_{h} \in X_{h}$ of the density (i.e. of the first component of $\left.\mathbf{w}^{\mathbf{k}}\right)$. Having $\rho_{h}\left(P_{i}\right)$ at each vertex of the triangulation, we can construct a piecewise linear recovery $c_{h}$ of the cost function $c$.

The sought potential $\phi_{h}$ is the fix point of the operator $\Lambda_{h}$, where $\Lambda_{h}: X_{h} \rightarrow X_{h}$ is the so called Hopf-Lax update function:

$$
\left(\Lambda_{h} \phi_{h}\right)\left(P_{i}\right)= \begin{cases}\min _{x \in \partial \mathscr{K}_{P_{i}}}\left(\phi_{h}(x)+\left\|P_{i}-x\right\| c_{h}\left(P_{i}\right)\right), & \text { where } P_{i} \in \Omega, \\ \phi_{h}\left(P_{i}\right) & P_{i} \in \partial \Omega .\end{cases}
$$

Here $\partial \mathscr{K}_{P_{i}}$ is the boundary of the set of volumes having the vertex $P_{i}$ in common. The details about the minimization process in Hopf-Lax update formula (34) and 
the convergence of the fix point iterations

$$
\phi_{h}^{n+1}=\Lambda_{h} \phi_{h}^{n}, n=0,1,2, \ldots, \text { with } \phi_{h}^{0}\left(P_{i}\right)= \begin{cases}\infty & P_{i} \in \Omega, \\ 0 & P_{i} \in \Gamma_{o}\end{cases}
$$

can be found in [Bornemann and Rasch 2006].

(2) Desired direction of velocity $\mu$ : Having computed the potencial $\phi_{h} \in X_{h}$ at each vertex of the mesh, we assess the gradient $\left.\nabla \phi_{h}\right|_{D_{i}}$ at the centre of gravity of the volume $D_{i}$ and $\mu$ is then given by relation (9).

(3) Discretization of equation (10): The following second order Runge-Kutta method is used for the discretization of equation (10)

$$
w_{i}^{k+1}=\frac{1}{2}\left(w_{i}^{k}+w^{(1)}+\tau_{k} s\left(w^{(1)}\right)\right),
$$

where

$$
w^{(1)}=w_{i}^{k}+\tau_{k} s\left(w_{i}^{k}\right)
$$

and the time step $\tau_{k}$ is one half of that given by the CFL condition for the considered finite volume method for system (11). The source term $s$ is given by relation (13), i.e. $s(w)=\left(0, \frac{1}{\tau} \rho(V(\rho) \mu(\rho)-v)\right)^{\mathrm{T}}$, the density $\rho$ being the first component of $w$.

(4) Finite volume solution of (11): For the solution of the homogenous equation (11) the MUSCL ${ }^{1}$ explicit finite volume method is employed with two stage TVD $^{2}$ Runge-Kutta time discretization, see e.g. [Feistauer et al. 2003]. The finite volume semi-discretization of (11) leads to a system of ordinary differential equations

$$
\frac{d w(t)}{d t}=\mathscr{H}(w(t)), w(0)=\mathbf{w}^{0},
$$

where the components of the vector valued function $\mathscr{H}$ are given by $H$ introduced in (23), namely $\mathscr{H}(w(t))=-\frac{1}{\left|D_{i}\right|} \sum_{j \in S(i)}\left|\Gamma_{i j}\right| H\left(w_{i}(t), w_{j}(t), n_{i j}\right) \quad$ with $w_{i}(t)=\int_{D_{i}} w(x, t) d x /\left|D_{i}\right|$. Its definition is an important issue - we use the analogy of (11) with the shallow water equations and propose the numerical flux of the Vijayasundaram type, which is derived in an analogy with that of shallow water equations. For comparison with the shallow water equations see [Felcman and Havle 2011]. The numerical solution of system (38) is based on the same

\footnotetext{
${ }^{1} \underline{\text { Monotonic U }}$ pstream-Centered $\underline{S}$ cheme for Conservation Laws

${ }^{2}$ Total aaiation Diminishing
} 


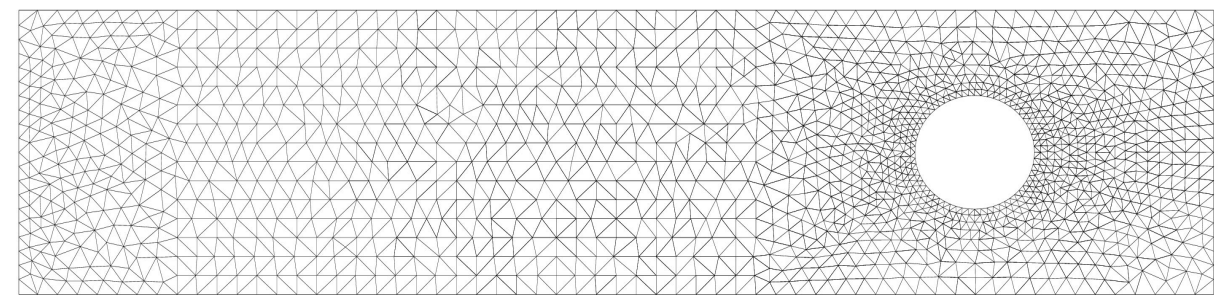

Fig. 1. Finite volume mesh with circular obstacle at point $[35,5]$ with radius $R=2 \mathrm{~m}$. We suppose outflow $\Gamma_{o}$ on the whole vertical right hand side and wall boundary $\Gamma_{w}$ elsewhere. Length of sides $40 \times 10 \mathrm{~m}$.

Runge-Kutta method and can be written in the form

$$
\mathbf{w}^{k+1}=\frac{1}{2}\left(\mathbf{w}^{k}+\mathbf{w}^{(1)}+\tau_{k} \mathscr{H}\left(\mathbf{w}^{(1)}, \mathscr{D}_{h}\right)\right)
$$

where

$$
\mathbf{w}^{(1)}=\mathbf{w}^{k}+\tau_{k} \mathscr{H}\left(\mathbf{w}^{k}, \mathscr{D}_{h}\right) .
$$

The time step $\tau_{k}$ is given by CFL stability condition stemming from the finite volume method. For the Runge-Kutta method, the time step is halved.

According to [Toro 1997] the complete splitting algorithm can be formally written in form:

$$
\mathbf{w}^{k+1}=\mathscr{S}\left(\tau_{k+1}, \mathscr{C}\left(\tau_{k}, \mathscr{S}\left(\tau_{k}, \mathbf{w}^{k}\right)\right)\right)
$$

where $\mathscr{C}$ is the operator for the solution of system (11), i.e. FVM step given by (39). By $\mathscr{S}$ we denote the operator for the solution of problem (10). In this operator we use the Bornemann and Rash algorithm for computing $\mu$ at first. Then the second order Runge-Kutta method, for the solution of system (10) with fixed $\mu$, is employed, for details see [Felcman and Kubera 2015].

\section{NUMERICAL EXPERIMENTS}

To show a practical application of the proposed numerical flux we suppose domain configurations as in Figure 1 with different obstacle radii $R \in[0,4]$. We study the dependency of the time of evacuation $T_{\text {evac }}$, i.e. the time in which all pedestrians are evacuated, on the radius of the obstacle and compare the results with those obtained the Lax/Friedrichs numerical flux. We use the following initial condition:

$$
\rho_{0}=\left\{\begin{array}{ll}
2 & x \in[0,20] \times[0,10], \\
0 & \text { elsewhere }
\end{array} \text { and } v_{0}=\mathbf{0} .\right.
$$




\begin{tabular}{|c|cc||c|cc|}
\hline Radius [m] & $T_{\text {evac }}^{\text {VS }[\mathrm{s}]}$ & $T_{\text {evac }}^{L F}[\mathrm{~s}]$ & Radius [m] & $T_{\text {evac }}^{V S}[\mathrm{~s}]$ & $T_{\text {evac }}^{L F}[\mathrm{~s}]$ \\
\hline \hline 0 & 30.4 & 33.8 & 2.25 & 39.7 & 39.6 \\
0.25 & 31.0 & 33.9 & 2.50 & 43.0 & 43.5 \\
0.50 & 31.4 & 34.2 & 2.75 & 47.0 & 44.0 \\
0.75 & 32.4 & 36.2 & 3.00 & 48.2 & 47.2 \\
1.00 & 32.6 & 37.1 & 3.25 & 54.6 & 51.4 \\
1.25 & 32.9 & 35.8 & 3.50 & 63.0 & 56.5 \\
1.50 & 34.6 & 36.0 & 3.75 & 66.0 & 62.9 \\
1.75 & 35.2 & 39.7 & 4.00 & 78.5 & 72.6 \\
2.00 & 37.3 & 37.8 & & & \\
\hline
\end{tabular}

Fig. 2. Comparison of computed $T_{\text {evac }}$ for different obstacle radius using the Vijayasundaram (VS) and Lax-Friedrichs (LF) numerical flux, repectively
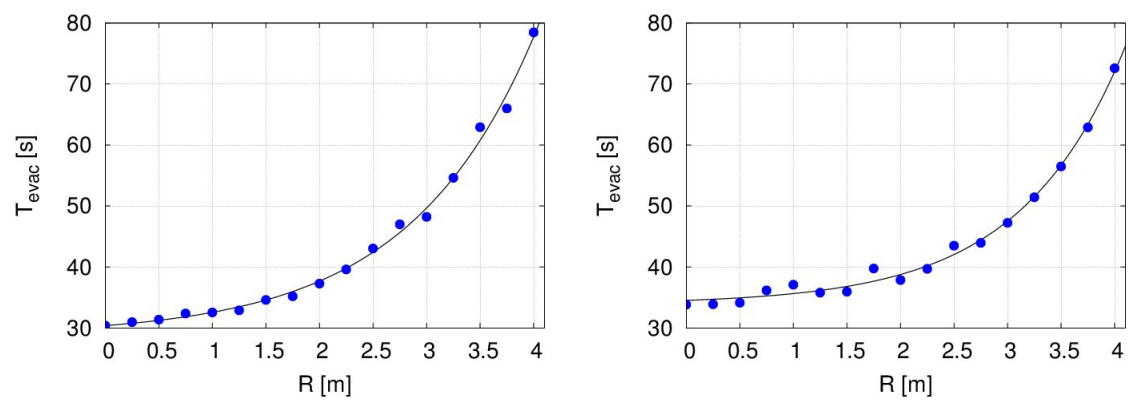

Fig. 3. The dependency of $T_{\text {evac }}$ on the radius of obstacle.

Vijayasundaram type numerical flux of (left), Lax-Friedrichs numerical flux (right)

The parameters of the model are: $p_{0}=1, \gamma=2$ in (3), $v_{\max }=2, \alpha=7.5, \rho_{\max }=9$ in (6) and $\tau=0.61$ in (10). These data are chosen from experimental investigation of pedestrian behaviour. For the experimental study see the real world investigation described in [Helbing et al. 2002]. We set the CFL number for the CFL condition $C F L=0.9$. We use the proposed numerical flux of Vijayasundaram type (VS) and compare obtained results with those obtained by the Lax-Friedrichs numerical flux (LF). The results for different obstacle radius for both numerical fluxes are summarized in Table 3 and depicted in Figure 3. The example of the distribution of density is depicted in Figure 4.

\section{CONCLUSION}

The proposed Vijayasundaram numerical flux for the pedestrian flow equations can be treated as competitive one to another numerical fluxes. The advantage can be its robustness proved for the Euler equations with respect to the mesh refinement and its applicability for an implicit version of the finite volume scheme followed by a suitable 

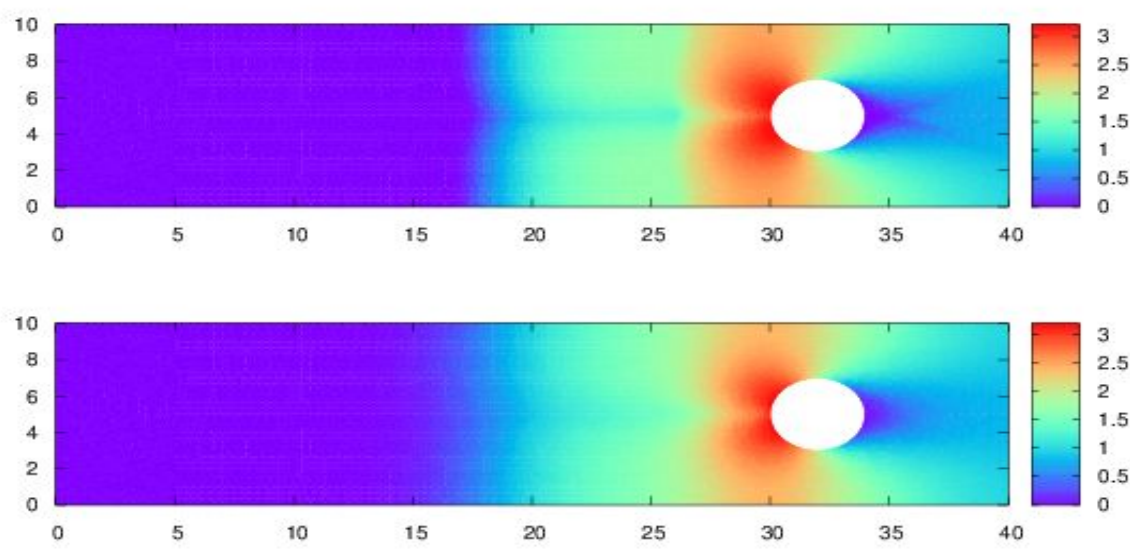

Fig. 4. Density distribution over the domain with circular obstacle at point $[32,5]$ with radius $R=2 \mathrm{~m}$ at time $t=15 \mathrm{~s}$. Vijayasundaram type numerical flux (up), Lax-Friedrichs numerical flux (down)

linearization.

We have also showed the ability of the splitting algorithm to solve a problem from the crowd dynamics. Next work will be devoted to the use of this algorithm in the framework of the shape optimization problems.

\section{ACKNOWLEDGEMENT}

The research of the first author was supported by the project Grant Severočeských dolů Chomutov, the research of the second author was supported by the project 13-00522S of the Czech Science Foundation. The authors acknowledge this support.

\section{REFERENCES}

Bellomo, N. And Dogbé, C. 2008. On the modelling crowd dynamics from scaling to hyperbolic macroscopic models. Mathematical Models and Methods in Applied Sciences 18, 1317 - 1345.

Bornemann, F. AND RASCH, C. 2006. Finite-element discretization of static hamilton-jacobi equations based on a local variational principle. Computing and Visualization in Science 9, 2, 57-69.

DRIDI, M. H. 2015. Simulation of high density pedestrian flow: Microscopic model. Open Journal of Modelling and Simulation 3, 1, $81-95$.

Eymard, R., Gallouët, T., And Herbin, R. 2000. Finite volume methods. In Handbook of Numerical Analysis, P. Ciarlet and J. Lions, Eds. Vol. VII. North-Holland, 713-1020.

Feistauer, M., Felcman, J., AND StrašKrabA, I. 2003. Mathematical and computational methods for compressible flow. Clarendon Press.

Felcman, J. And HaVle, O. 2011. On a numerical flux for the shallow water equations. Applied Mathematics and Computation 217, 11, 5160 - 5170. Special issue on Fluid Flow and Heat Transfer. 
Felcman, J. And Kubera, P. 2015. Modeling pedestrian flow via shallow water equations. (in preparation).

FurmaneK, P., Furst, J., And Kozel, K. 2009. High order finite volume schemes for numerical solution of 2D and 3D transonic flows. KYBERNETIKA 45, 4, 567-579.

Gallouët, T., Hérard, J.-M., AND Seguin, N. 2003. Some approximate godunov schemes to compute shallow-water equations with topography. Comput. Fluids 32, 4, 479-513.

Helbing, D., FArkas, I. J., Molnár, P., And ViCSEK, T. 2002. Simulation of pedestrian crowd in normal and evacuation situations. Pedestrian and Evacuation Dynamics Journal, 21 - 58.

MARNO, P. 2002. Crowded - macroscopic and microscopic models for pedestrian dynamics. Ph.D. thesis, University of Reading.

Toro, E. F. 1997. Riemann Solvers and Numerical Methods for Fluid Dynamics. Springer, Berlin.

Twarogowska, M., Goatin, P., And Duvigneau, R. 2013. Numerical study of macroscopic pedestrian flow models. Tech. rep.

VijayAsundaram, G. 1982. Resolution numérique des équations d'euler pour des écoulements transsoniques avec un schéma de godunov en éléments finis. Ph.D. thesis, Paris IV.

\author{
Petr Kubera \\ Jan Evengelista Purkyně University \\ Faculty of Science, Department of Informatics \\ and \\ Czech Technical University in Prague \\ Faculty of Nuclear Sciences and Physical Engineering \\ Department of Informatics \\ email: kubera.petr@gmail.com
}

Jiří Felcman

Charles University in Prague

Faculty of Mathematics and Physics

Department of Numerical Mathematics

email: felcman@karlin.mff.cuni.cz 